\title{
Bilevel minimax theorems for non-continuous set-valued mappings
}

\section{Yen-Cherng Lin*}

\section{${ }^{\text {*Correspondence: }}$}

yclin@mail.cmu.edu.tw

Department of Occupational Safety

and Health, College of Public

Health, China Medical University,

Taichung, 40421, Taiwan

\begin{abstract}
We study new types for minimax theorems with a couple of set-valued mappings, and we propose several versions for minimax theorems in topological vector spaces setting. These problems arise naturally from some minimax theorems in the vector settings. Both the types of scalar minimax theorems and the set minimax theorems are discussed. Furthermore, we propose three versions of minimax theorems for the last type. Some examples are also proposed to illustrate our theorems.
\end{abstract}

MSC: 49J35; 58C06

Keywords: minimax theorems; cone-convexities

\section{Introduction and preliminaries}

Let $X, Y$ be two nonempty sets in two Hausdorff topological vector spaces, respectively, $Z$ be a Hausdorff topological vector space, $C \subset Z$ a closed convex and pointed cone with apex at the origin and int $C \neq \emptyset$, this means that $C$ is a closed set with nonempty interior and satisfies $\lambda C \subseteq C, \forall \lambda>0 ; C+C \subseteq C$; and $C \cap(-C)=\{0\}$. The scalar bilevel minimax theorems stated as follows: given two set-valued mappings $F, G: X \times Y \rightrightarrows \mathbb{R}$, under suitable conditions the following relation holds:

$$
(s-B) \quad \min \bigcup_{x \in X} \max \bigcup_{y \in Y} F(x, y) \leq \max \bigcup_{y \in Y} \min \bigcup_{x \in X} G(x, y) .
$$

Given two mappings $F, G: X \times Y \rightrightarrows Z$, the first version of bilevel minimax theorems stated that under suitable conditions the following relation holds:

$\left(B_{1}\right) \quad \operatorname{Max} \bigcup_{y \in Y} \operatorname{Min}_{w} \bigcup_{x \in X} G(x, y) \subset \operatorname{Min}\left(\operatorname{co\bigcup } \bigcup_{x \in X} \operatorname{Max}_{w} \bigcup_{y \in Y} F(x, y)\right)+C$.

The second version of bilevel minimax theorems stated that under suitable conditions the following relation holds:

$\left(B_{2}\right) \quad \operatorname{Max} \bigcup_{y \in Y} \operatorname{Min}_{w} \bigcup_{x \in X} G(x, y) \subset \operatorname{Min}_{x \in X} \operatorname{Max}_{w} \bigcup_{y \in Y} F(x, y)+C$.

O2014 Lin; licensee Springer. This is an Open Access article distributed under the terms of the Creative Commons Attribution License (http://creativecommons.org/licenses/by/2.0), which permits unrestricted use, distribution, and reproduction in any medium, provided the original work is properly cited. 
The third version of bilevel minimax theorems stated that under suitable conditions the following relation holds:

$\left(B_{3}\right) \quad \operatorname{Min} \bigcup_{x \in X} \operatorname{Max}_{w} \bigcup_{y \in Y} F(x, y) \subset \operatorname{Max} \bigcup_{y \in Y} \operatorname{Min}_{w} \bigcup_{x \in X} G(x, y)+Z \backslash(C \backslash\{0\})$.

The case $G=F$ of $(s-B)$ and $\left(B_{1}\right)-\left(B_{3}\right)$ has been discussed in [1-3] for set-valued mapping and in [4] for vector-valued mapping, respectively. Scalar minimax theorems and set minimax theorems for non-continuous set-valued mappings were first proposed by Lin et $a l$. [1]. These results can be compared with the recent existing results [2, 3]. In this paper, we establish bilevel minimax results with a couple of non-continuous set-valued mappings (Theorem 2.1 in Section 2, Theorems 3.1-3.3 in Section 3). These results might not hold for each individual non-continuous set-valued mapping since it always lack some conditions so that the existing minimax theorems are not applicable, such as Theorems 4.1-4.3 [1], Theorem 2.1 [2] or Proposition 2.1 [3].

We present some fundamental concepts which will be used in the sequel.

Definition 1.1 [1, 2, 4] Let $A$ be a nonempty subset of $Z$. A point $z \in A$ is called a

(a) minimal point of $A$ if $A \cap(z-C)=\{z\}$; $\operatorname{Min} A$ denotes the set of all minimal points of $A$;

(b) maximal point of $A$ if $A \cap(z+C)=\{z\}$; Max $A$ denotes the set of all maximal points of $A$;

(c) weakly minimal point of $A$ if $A \cap(z-\operatorname{int} C)=\emptyset ; \operatorname{Min}_{w} A$ denotes the set of all weakly minimal points of $A$;

(d) weakly maximal point of $A$ if $A \cap(z+\operatorname{int} C)=\emptyset ; \operatorname{Max}_{w} A$ denotes the set of all weakly maximal points of $A$.

Following [2], we denote both Max and $\operatorname{Max}_{w}$ by max (both Min and $\operatorname{Min}_{w}$ by min) in $\mathbb{R}$ since both Max and $\operatorname{Max}_{w}$ (both Min and $\operatorname{Min}_{w}$ ) are same in $\mathbb{R}$. We note that, for a nonempty compact set $A$, the both sets $\operatorname{Max} A$ and $\operatorname{Min} A$ are nonempty. Furthermore, $\operatorname{Min} A \subset \operatorname{Min}_{w} A, \operatorname{Max} A \subset \operatorname{Max}_{w} A, A \subset \operatorname{Min} A+C$, and $A \subset \operatorname{Max} A-C$.

In the sequel we shall use the following geometric result.

Lemma 1.1 [5] Let $X, Y$ be nonempty convex subsets of two real Hausdorff topological spaces, respectively, $A \subset X \times Y$ be a subset such that

(a) for each $y \in Y$, the set $\{x \in X:(x, y) \in A\}$ is closed in $X$; and

(b) for each $x \in X$, the set $\{y \in Y:(x, y) \notin A\}$ is convex or empty.

Suppose that there exist a subset $B$ of $A$ and a compact convex subset $K$ of $X$ such that $B$ is closed in $X \times Y$ and

(c) for each $y \in Y$, the set $\{x \in K:(x, y) \in B\}$ is nonempty and convex.

Then there exists a point $x_{0} \in K$ such that $\left\{x_{0}\right\} \times Y \subset A$.

Definition 1.2 Let $U, V$ be Hausdorff topological spaces. A set-valued map $F: U \rightrightarrows V$ with nonempty values is said to be

(a) lower semi-continuous at $x_{0} \in U$ if for any net $\left\{x_{\mu}\right\} \subset U$ such that $x_{\mu} \rightarrow x_{0}$ and any $y_{0} \in F\left(x_{0}\right)$, there exists a net $y_{\mu} \in F\left(x_{\mu}\right)$ such that $y_{\mu} \rightarrow y_{0}$; 
(b) upper semi-continuous at $x_{0} \in U$ if for every $x_{0} \in U$ and for every open set $N$ containing $F\left(x_{0}\right)$, there exists a neighborhood $M$ of $x_{0}$ such that $F(M) \subset N$;

(c) continuous at $x_{0} \in U$ if $F$ is upper semi-continuous as well as lower semi-continuous at $x_{0}$.

We note that $T$ is upper semi-continuous at $x_{0}$ and $T\left(x_{0}\right)$ is compact, then for any net $\left\{x_{v}\right\} \subset U, x_{v} \rightarrow x_{0}$, and for any net $y_{v} \in T\left(x_{v}\right)$ for each $v$, there exist $y_{0} \in T\left(x_{0}\right)$ and a subnet $\left\{y_{v_{\alpha}}\right\}$ such that $y_{v_{\alpha}} \rightarrow y_{0}$. For more details, we refer the reader to $[6,7]$.

Definition $1.3[2,8]$ Let $k \in \operatorname{int} C$ and $v \in Z$. The Gerstewitz function $\xi_{k v}: Z \rightarrow \mathbb{R}$ is defined by

$$
\xi_{k v}(u)=\min \{t \in \mathbb{R}: u \in v+t k-C\} .
$$

We present some fundamental properties of the scalarization function.

Proposition 1.1 $[2,8]$ Let $k \in$ int $C$ and $v \in Z$. The Gerstewitz function $\xi_{k v}: Z \rightarrow \mathbb{R}$ has the following properties:

(a) $\xi_{k v}(u) \leq r \Leftrightarrow u \in v+r k-C$;

(b) $\xi_{k v}(u)<r \Leftrightarrow u \in v+r k-\operatorname{int} C$; and

(c) $\xi_{k v}(\cdot)$ is a continuous convex increasing and strictly increasing function.

We also need the following different kinds of cone-convexities for set-valued mappings.

Definition 1.4 [1] Let $X$ be a nonempty convex subset of a topological vector space. A setvalued mapping $F: X \rightrightarrows Z$ is said to be

(a) above-C-convex (respectively, above-C-concave) on $X$ if for all $x_{1}, x_{2} \in X$ and all $\lambda \in[0,1]$,

$$
\begin{aligned}
& F\left(\lambda x_{1}+(1-\lambda) x_{2}\right) \subset \lambda F\left(x_{1}\right)+(1-\lambda) F\left(x_{2}\right)-C \\
& \left(\text { respectively, } \lambda F\left(x_{1}\right)+(1-\lambda) F\left(x_{2}\right) \subset F\left(\lambda x_{1}+(1-\lambda) x_{2}\right)-C\right)
\end{aligned}
$$

(b) above-naturally $C$-quasi-convex on $X$ if for all $x_{1}, x_{2} \in X$ and all $\lambda \in[0,1]$,

$$
F\left(\lambda x_{1}+(1-\lambda) x_{2}\right) \subset \operatorname{co}\left\{F\left(x_{1}\right) \cup F\left(x_{2}\right)\right\}-C,
$$

where $\operatorname{co} A$ denotes the convex hull of a set $A$;

Let $C^{\star}=\left\{g \in Z^{\star}: g(c) \geq 0\right.$ for all $\left.c \in C\right\}$, where $Z^{\star}$ is the set of all nonzero continuous linear functional on $Z$.

Proposition 1.2 Let $A$ be a nonempty compact subset of $Z$, for any $\xi \in C^{\star}$, we have $\xi \operatorname{Max}_{w} A \subset \max \xi A-\mathbb{R}_{+}$and $\max \xi A \in \xi \operatorname{Max}_{w} A-\mathbb{R}_{+}$.

Proof $\max \xi A$ exists since $\xi A$ is compact. There is $u \in A$ such that $\xi u=\max \xi A$. By the Proposition 3.14 of [1], we have $u \in \operatorname{Max}_{w} A$. Thus, $\max \xi A \in \xi\left(\operatorname{Max}_{w} A\right) \subset \xi\left(\operatorname{Max}_{w} A\right)-$ $\mathbb{R}_{+}$. Furthermore, for any $t \in \xi \operatorname{Max}_{w} A$, there exists $u \in \operatorname{Max}_{w} A \subset A$ such that $t=\xi u \leq$ $\max \xi A$. Thus, $\xi \operatorname{Max}_{w} A \subset \max \xi A-\mathbb{R}_{+}$. 
By using a similar argument as in Proposition 1.2, we can deduce the following conclusion.

Proposition 1.3 Let $A$ be a nonempty compact subset of $Z$, for any $\xi \in C^{\star}$, we have $\xi \operatorname{Min}_{w} A \subset \min \xi A+\mathbb{R}_{+}$and $\min \xi A \subset \xi \operatorname{Min}_{w} A+\mathbb{R}_{+}$.

The following proposition can be derived from Definition 1.1 and Proposition 1.1, so we omit the proof.

Proposition 1.4 Suppose that $\bigcup_{x \in X} F(x)$ is compact. For any given $k \in \operatorname{int} C, v \in Z$ and a Gerstewitz function $\xi_{k v}: Z \rightarrow \mathbb{R}$. Then, for any $d \in \operatorname{Min}_{w} \bigcup_{x \in X} F(x)$, we have $\xi_{k v} d \in \min \bigcup_{x \in X} \xi_{k v} F(x)+\mathbb{R}_{+}$. Similarly, for any $d \in \operatorname{Max}_{w} \bigcup_{x \in X} F(x)$, we have $\xi_{k v} d \in$ $\max \bigcup_{x \in X} \xi_{k v} F(x)-\mathbb{R}_{+}$.

We note that, if $X$ is nonempty compact set and $F: X \rightrightarrows Z$ is upper semi-continuous with nonempty compact values, then Proposition 1.4 is also valid.

Proposition 1.5 If $x \mapsto G(x, y)$ is above-naturally $C$-quasi-convex on $X$ for each $y \in Y$, and a Gerstewitz function $\xi_{k v}: Z \rightarrow \mathbb{R}$, then $x \mapsto \xi_{k v} G(x, y)$ is above-naturally $\mathbb{R}_{+}$-quasi-convex on $X$ for each $y \in Y$.

In the proof of Proposition 1.5, we need to use the monotonicity and positive homogeneous property of $\xi_{k v}$, and a similar technique of Proposition 3.13 [1], we leave the readers to prove it.

\section{Scalar bilevel minimax theorems}

We first establish the following scalar bilevel minimax theorem.

Theorem 2.1 Let $X, Y$ be two nonempty compact convex subsets of real Hausdorff topological vector spaces, respectively. The set-valued mappings $F, G: X \times Y \rightrightarrows \mathbb{R}$ with $F(x, y) \subset$ $G(x, y)$ such that the sets $\bigcup_{y \in Y} F(x, y), \bigcup_{x \in X} G(x, y)$ and $G(x, y)$ are compact for all $(x, y) \in$ $X \times Y$, and they satisfy the following conditions:

(i) $x \mapsto F(x, y)$ is lower semi-continuous on $X$ for each $y \in Y$ and $y \mapsto F(x, y)$ is above- $\mathbb{R}_{+}$-concave on $Y$ for each $x \in X$

(ii) $x \mapsto G(x, y)$ is above-naturally $\mathbb{R}_{+}$-quasi-convex for each $y \in Y$, and $(x, y) \mapsto G(x, y)$ is lower semi-continuous on $X \times Y$; and

(iii) for each $w \in Y$, there is an $x_{w} \in X$ such that

$$
\max G\left(x_{w}, w\right) \leq \max \bigcup_{y \in Y} \min \bigcup_{x \in X} G(x, y)
$$

Then the relation $(s-B)$ holds.

Proof For each $y \in Y$, the compactness of $\bigcup_{x \in X} G(x, y)$ implies the existence of $\min \bigcup_{x \in X} G(x, y)$. By the lower semi-continuity of $G$ and Lemma 3.1 [9], the mapping $y \mapsto \bigcup_{x \in X} G(x, y)$ is lower semi-continuous with nonempty compact values. By Lemma 3.2 [9], the mapping $y \mapsto \min \bigcup_{x \in X} G(x, y)$ is upper semi-continuous function on $Y$. Since $Y$ is nonempty and compact, the set $\bigcup_{y \in Y} \min \bigcup_{x \in X} G(x, y)$ is nonempty and compact. This 
implies that the maximal points of $\bigcup_{y \in Y} \min \bigcup_{x \in X} G(x, y)$ exist. Another similar argument to explain the left-hand side of $(s-B)$ exists. Therefore, both sides of the relation $(s-B)$ make sense.

For any given $t \in \mathbb{R}$ with $t>\max \bigcup_{y \in Y} \min \bigcup_{x \in X} G(x, y)$. Define two sets $A, B \subset X \times Y$ by

$$
A=\{(x, y) \in X \times Y: \forall f \in F(x, y), f \leq t\}
$$

and

$$
B=\{(x, y) \in X \times Y: \forall g \in G(x, y), g \leq t\} .
$$

Since $F(x, y) \subset G(x, y)$ for all $(x, y) \in X \times Y$, we have

$$
\emptyset \neq B \subset A .
$$

The nonempty property of $B$ can be deduced from the choice of $t$ and (iii).

Choose any $y_{1}, y_{2} \in Y \backslash A(x)=\{y \in Y: \exists f \in F(x, y), f>t\}$. There exist $f_{1} \in F\left(x, y_{1}\right)$ with $f_{1}>t$ and $f_{2} \in F\left(x, y_{2}\right)$ with $f_{2}>t$. Then, for any $\lambda \in[0,1], t \in \lambda F\left(x, y_{1}\right)+(1-\lambda) F\left(x, y_{2}\right)-\mathbb{R}_{+}$. By the above- $\mathbb{R}_{+}$-concavity of $F$, we see that there is $f_{\lambda} \in F\left(x, \lambda y_{1}+(1-\lambda) y_{2}\right)$ such that $f_{\lambda}>t$. Thus, $\lambda y_{1}+(1-\lambda) y_{2} \in Y \backslash A(x)$, and hence $Y \backslash A(x)$ is convex for each $x \in X$. Similarly, by the above-naturally $\mathbb{R}_{+}$-convexity of $G$, the set $\{x \in X:(x, y) \in B\}$ is convex for each $y \in Y$. Furthermore, by the lower semi-continuity of $G$, we know that the set $B$ is closed.

Since all conditions of Lemma 1.1 hold, by Lemma 1.1, there exists a point $x_{0} \in X$ such that $\left\{x_{0}\right\} \times Y \subset A$, that is, there exists a point $x_{0} \in X$ such that

$$
\forall f \in F\left(x_{0}, y\right), \quad f \leq t,
$$

for all $y \in Y$. Thus, we know that $\max \bigcup_{y \in Y} F\left(x_{0}, y\right) \leq t$ and the relation $(s-B)$ is valid.

We see that Theorem 2.1 includes the case $G=F$ as a special case. We state the following.

Corollary 2.1 Let $X, Y$ be two nonempty compact convex subsets of real Hausdorff topological vector spaces, respectively. The set-valued mapping $F: X \times Y \rightrightarrows \mathbb{R}$ such that the sets $\bigcup_{y \in Y} F(x, y), \bigcup_{x \in X} F(x, y)$ and $F(x, y)$ are compact for all $(x, y) \in X \times Y$, and they satisfy the following conditions:

(i) $y \mapsto F(x, y)$ is above- $\mathbb{R}_{+}$-concave on $Y$ for each $x \in X$;

(ii) $x \mapsto F(x, y)$ is above-naturally $\mathbb{R}_{+}$-quasi-convex for each $y \in Y$, and $(x, y) \mapsto F(x, y)$ is lower semi-continuous on $X \times Y$; and

(iii) for each $w \in Y$, there is an $x_{w} \in X$ such that

$$
\max F\left(x_{w}, w\right) \leq \max \bigcup_{y \in Y} \min \bigcup_{x \in X} F(x, y) .
$$

Then we have the relation $(s-B)$ with $G=F$ holds. 
If, in additional, the mapping $(x, y) \mapsto F(x, y)$ is upper semi-continuous with nonempty compact values on $X \times Y$ in Corollary 2.1, then we can easy see that the both sets $\bigcup_{y \in Y} F(x, y)$ and $\bigcup_{x \in X} F(x, y)$ are compact. Hence we can deduce the following result due to Li et al. ([3, Proposition 2.1]).

Corollary 2.2 Let $X, Y$ be two nonempty compact convex subsets of real Hausdorff topological vector spaces, respectively. The set-valued mapping $F: X \times Y \rightrightarrows \mathbb{R}$ is continuous with nonempty compact values and satisfies the following conditions:

(i) $y \mapsto F(x, y)$ is above- $\mathbb{R}_{+}$-concave on $Y$ for each $x \in X$;

(ii) $x \mapsto F(x, y)$ is above-naturally $\mathbb{R}_{+}$-quasi-convex for each $y \in Y$; and

(iii) for each $y \in Y$, there is an $x_{y} \in X$ such that

$$
\max F\left(x_{y}, y\right) \leq \max \bigcup_{y \in Y} \min \bigcup_{x \in X} F(x, y)
$$

Then we have the relation $(s-B)$ with $G=F$ holds.

Throughout the rest of this paper, we assume that $X, Y$ are two nonempty compact convex subsets of real Hausdorff topological vector spaces, respectively, and $Z$ is a complete locally convex Hausdorff topological vector space.

\section{The bilevel minimax theorems}

In this section, we will present three versions of bilevel minimax theorems. As the following result illustrates, the relation $\left(B_{1}\right)$ is true.

Theorem 3.1 Suppose that the set-valued mappings $F, G: X \times Y \rightrightarrows Z$ with $F(x, y) \subset$ $G(x, y)$ for all $(x, y) \in X \times Y$, and they satisfy the following conditions:

(i) the mapping $(x, y) \mapsto F(x, y)$ is upper semi-continuous with nonempty compact values, the mapping $x \mapsto F(x, y)$ is lower semi-continuous for each $y \in Y$, and $y \mapsto F(x, y)$ is above-C-concave on $Y$ for each $x \in X$;

(ii) $x \mapsto G(x, y)$ is above-naturally C-quasi-convex for each $y \in Y,(x, y) \mapsto G(x, y)$ is continuous with nonempty compact values on $X \times Y$;

(iii) for each $w \in Y$ and for each $\xi \in C^{\star}$, there is an $x_{w} \in X$ such that

$$
\max \xi G\left(x_{w}, w\right) \leq \max \bigcup_{y \in Y} \min \bigcup_{x \in X} \xi G(x, y)
$$

and

(iv) for each $w \in Y$,

$$
\operatorname{Max} \bigcup_{y \in Y} \operatorname{Min}_{w} \bigcup_{x \in X} G(x, y) \subset \operatorname{Min}_{w} \bigcup_{x \in X} G(x, w)+C .
$$

Then the relation $\left(B_{1}\right)$ is valid.

Proof Let $\Lambda(x):=\operatorname{Max}_{w} \bigcup_{y \in Y} F(x, y)$ for all $x \in X$. From Lemma 2.4 [1] and Proposition 3.5 [1], the mapping $x \mapsto \Lambda(x)$ is upper semi-continuous with nonempty compact values on $X$. Hence $\bigcup_{x \in X} \Lambda(x)$ is compact, and so is $\operatorname{co}\left(\bigcup_{x \in X} \Lambda(x)\right)$. Then $\operatorname{co}\left(\bigcup_{x \in X} \Lambda(x)\right)+C$ is closed 
convex set with nonempty interior. Suppose that $v \notin \operatorname{co}\left(\bigcup_{x \in X} \Lambda(x)\right)+C$. By separation theorem, there is a $k \in \mathbb{R}, \epsilon>0$ and a nonzero continuous linear functional $\xi: Z \mapsto \mathbb{R}$ such that

$$
\xi(v) \leq k-\epsilon<k \leq \xi(u+c)
$$

for all $u \in \operatorname{co}\left(\bigcup_{x \in X} \Lambda(x)\right)$ and $c \in C$. From this we can see that $\xi \in C^{\star}$ and

$$
\xi(v)<\xi(u)
$$

for all $u \in \operatorname{co}\left(\bigcup_{x \in X} \Lambda(x)\right)$. By Proposition 3.14 of [1], for any $x \in X$, there is a $y_{x}^{\star} \in Y$ and $f\left(x, y_{x}^{\star}\right) \in F\left(x, y_{x}^{\star}\right)$ with $f\left(x, y_{x}^{\star}\right) \in \Lambda(x)$ such that

$$
\xi f\left(x, y_{x}^{\star}\right)=\max \bigcup_{y \in Y} \xi F(x, y)
$$

Let us choose $c=0$ and $u=f\left(x, y_{x}^{\star}\right)$ in equation (1), we have

$$
\xi(v)<\xi\left(f\left(x, y_{x}^{\star}\right)\right)=\max \bigcup_{y \in Y} \xi F(x, y)
$$

for all $x \in X$. Therefore,

$$
\xi(v)<\min \bigcup_{x \in X} \max \bigcup_{y \in Y} \xi F(x, y)
$$

From conditions (i)-(iii), applying Proposition 3.9 and Proposition 3.13 in [1], all conditions of Theorem 2.1 hold. Hence we have

$$
\xi(v)<\max \bigcup_{y \in Y} \min \bigcup_{x \in X} \xi G(x, y) .
$$

Since $Y$ is compact, there is a $y^{\prime} \in Y$ such that

$$
\xi(v)<\min \bigcup_{x \in X} \xi G\left(x, y^{\prime}\right) .
$$

Thus,

$$
v \notin \bigcup_{x \in X} G\left(x, y^{\prime}\right)+C,
$$

and hence

$$
v \notin \operatorname{Min}_{w} \bigcup_{x \in X} G\left(x, y^{\prime}\right)+C .
$$

By (iv) and (2), we have

$$
v \notin \operatorname{Max} \bigcup_{y \in Y} \operatorname{Min}_{w} \bigcup_{x \in X} G(x, y) .
$$


Hence, for every $v \in \operatorname{Max} \bigcup_{y \in Y} \operatorname{Min}_{w} \bigcup_{x \in X} G(x, y)$, we have

$$
v \in \operatorname{co}\left(\bigcup_{x \in X} \Lambda(x)\right)+C
$$

That is, the relation $\left(B_{1}\right)$ is valid.

Remark 3.1 We note that Theorem 3.1 includes the case $G=F$ as a special case, and it almost can be compared with Theorem 3.1 [2]. Neither $F$ nor $G$ is able to apply the theorems in $[2,3]$ to deduce the minimax properties since $F$ is not continuous and $G$ does not satisfy the conditions (iv)-(v) of Theorem 3.1 [2], $\left(H_{1}\right)-\left(H_{2}\right)$ of Theorem 3.1 [3] or $\left(H_{3}\right)$ $\left(H_{4}\right)$ of Theorem 3.2 [3].

We note that the relation $\left(B_{1}\right)$ does not hold for any two mappings satisfy the condition $F(x, y) \subset G(x, y)$ for all $(x, y) \in X \times Y$, even though both of $F$ and $G$ are continuous setvalued mappings. For example, let $F(x, y)=\{x\} \times\left[1-\sqrt{1-y^{2}}, 1+\sqrt{1-y^{2}}\right]$ and $G(x, y)=$ $\{x\} \times\left[-1,1+\sqrt{1-y^{2}}\right]$ for all $x, y \in[-1,1]=X=Y$. Hence we propose the following example to illustrate the validity of Theorem 3.1 .

Example 3.1 Let $X=[0,1], Y=[-1,0], Z=\mathbb{R}^{2}$ and $C=C^{\star}=\mathbb{R}_{+}^{2}$. Define $H: X \rightrightarrows X$ by

$$
H(y)= \begin{cases}{[-1,0],} & y=-1 \\ \{0\}, & y \neq-1\end{cases}
$$

Define $F, G: X \times Y \mapsto Z$ by

$$
F(x, y)=\left\{x^{2}\right\} \times H(y)
$$

and

$$
G(x, y)=\left[0, x^{2}\right] \times[y, 0]
$$

for all $(x, y) \in X \times Y$. We can see that $F(x, y) \subset G(x, y)$ for all $(x, y) \in X \times Y$, and conditions (i)-(ii) of Theorem 3.1 hold. We now claim that the condition (iii) of Theorem 3.1 is valid. Indeed, for each $\xi=\left(\xi_{1}, \xi_{2}\right) \in C^{\star}$, since

$$
\begin{aligned}
\max \xi G(x, y) & =\max \left\{\xi_{1} s+\xi_{2} t: 0 \leq s \leq x^{2}, y \leq t \leq 0\right\} \\
& =\xi_{1} x^{2}
\end{aligned}
$$

for all $(x, y) \in X \times Y$, and

$$
\min \bigcup_{x \in[0,1]} \xi G(x, y)=\xi_{2} y
$$

for all $y \in Y$, we have

$$
\max \bigcup_{y \in[-1,0]} \min \bigcup_{x \in[0,1]} \xi G(x, y)=0
$$


For each $w \in Y$, we can choose $x_{w}=0$ such that the condition (iii) is valid. The reason so that the condition (iv) is valid can be explained as follows: for each $w \in Y$, we see that

$$
\operatorname{Min}_{w} \bigcup_{x \in X} G(x, w)=(\{0\} \times[w, 0]) \cup([0,1] \times\{w\})
$$

and

$$
\operatorname{Max} \bigcup_{y \in Y} \operatorname{Min}_{w} \bigcup_{x \in X} G(x, y)=\{(1,0)\} .
$$

This implies that

$$
\operatorname{Max} \bigcup_{y \in Y} \operatorname{Min}_{w} \bigcup_{x \in X} G(x, y) \subset \operatorname{Min}_{w} \bigcup_{x \in X} G(x, w)+C,
$$

and so the condition (iv) holds. Finally, from the observation of

$$
\operatorname{Min}\left(\operatorname{co} \bigcup_{x \in X} \operatorname{Max}_{w} \bigcup_{y \in Y} F(x, y)\right)=\{(0,-1)\}
$$

the relation $\left(B_{1}\right)$ is valid.

Corollary 3.1 Suppose that the set-valued mapping $F: X \times Y \rightrightarrows Z$ such that the following conditions are satisfied:

(i) the mapping $(x, y) \mapsto F(x, y)$ is continuous with nonempty compact values, and $y \mapsto F(x, y)$ is above-C-concave on $Y$ for each $x \in X$;

(ii) $x \mapsto F(x, y)$ is above-naturally $C$-quasi-convex for each $y \in Y$;

(iii) for each $w \in Y$ and for each $\xi \in C^{\star}$, there is an $x_{w} \in X$ such that

$$
\max \xi F\left(x_{w}, w\right) \leq \max \bigcup_{y \in Y} \min \bigcup_{x \in X} \xi F(x, y)
$$

and

(iv) for each $w \in Y$,

$$
\operatorname{Max} \bigcup_{y \in Y} \operatorname{Min}_{w} \bigcup_{x \in X} F(x, y) \subset \operatorname{Min}_{w} \bigcup_{x \in X} F(x, w)+C .
$$

Then the relation $\left(B_{1}\right)$ with $G=F$ is valid.

In the following result, we apply the Gerstewitz function $\xi_{k v}: Z \mapsto \mathbb{R}$ to introduce the second version of bilevel minimax theorems, where $k \in \operatorname{int} C$ and $v \in Z$.

Theorem 3.2 Suppose that the set-valued mappings $F, G: X \times Y \rightrightarrows Z$ such that $F(x, y) \subset$ $G(x, y)$ for all $(x, y) \in X \times Y$, and the following conditions are satisfied:

(i) the mapping $(x, y) \mapsto F(x, y)$ is upper semi-continuous with nonempty compact values, the mapping $x \mapsto F(x, y)$ is lower semi-continuous for all $y \in Y$; 
(ii) $x \mapsto G(x, y)$ is above-naturally $C$-quasi-convex on $X$ for each $y \in Y,(x, y) \mapsto G(x, y)$ is continuous with nonempty compact values on $X \times Y$;

(iii) given any Gerstewitz function $\xi_{k v}$ with $v \notin \bigcup_{x \in X} \operatorname{Max}_{w} \bigcup_{y \in Y} F(x, y)+C$ satisfies the following conditions:

(iii $\left.{ }_{\mathrm{a}}\right) y \mapsto \xi_{k v} F(x, y)$ is above- $\mathbb{R}_{+}$-concave for all $x \in X$; and

(iii $\left.{ }_{\mathrm{b}}\right)$ for each $w \in Y$, there is an $x_{w} \in X$ such that

$$
\max \xi_{k v} G\left(x_{w}, w\right) \leq \max \bigcup_{y \in Y} \min \bigcup_{x \in X} \xi_{k v} G(x, y) ; \quad \text { and }
$$

(iv) for each $w \in Y$,

$$
\operatorname{Max} \bigcup_{y \in Y} \operatorname{Min}_{w} \bigcup_{x \in X} G(x, y) \subset \operatorname{Min}_{w} \bigcup_{x \in X} G(x, w)+C
$$

Then the relation $\left(B_{2}\right)$ is valid.

Proof Let $\Lambda(x)$ be defined in the same way as in Theorem 3.1 for all $x \in X$. Using the same process in the proof of Theorem 3.1, we know that the set $\bigcup_{x \in X} \Lambda(x)$ is nonempty and compact. For any $v \notin \bigcup_{x \in X} \Lambda(x)+C$, there is a Gerstewitz function $\xi_{k v}: Z \mapsto \mathbb{R}$ with some $k \in$ int $C$ such that

$$
\xi_{k v}(u)>0
$$

for all $u \in \bigcup_{x \in X} \Lambda(x)$. Then, for each $x \in X$, there is $y_{x}^{\star} \in Y$ and $f\left(x, y_{x}^{\star}\right) \in F\left(x, y_{x}^{\star}\right)$ with $f\left(x, y_{x}^{\star}\right) \in \operatorname{Max}_{w} \bigcup_{y \in Y} F(x, y)$ such that

$$
\xi_{k v}\left(f\left(x, y_{x}^{\star}\right)\right)=\max \bigcup_{y \in Y} \xi_{k v} F(x, y) .
$$

Choosing $u=f\left(x, y_{x}^{\star}\right)$ in equation (3), we have

$$
\max \bigcup_{y \in Y} \xi_{k v} F(x, y)>0
$$

for all $x \in X$. Therefore,

$$
\min \bigcup_{x \in X} \max \bigcup_{y \in Y} \xi_{k v} F(x, y)>0
$$

By Proposition 1.5 and combining conditions (i)-(iii), we know that all conditions of Theorem 2.1 hold, and by relation $(s-B)$ we have

$$
\max \bigcup_{y \in Y} \min \bigcup_{x \in X} \xi_{k \nu} G(x, y)>0
$$

Since $Y$ is compact, there is a $y^{\prime} \in Y$ such that

$$
\min \bigcup_{x \in X} \xi_{k \nu} G\left(x, y^{\prime}\right)>0
$$


Thus,

$$
v \notin \bigcup_{x \in X} G\left(x, y^{\prime}\right)+C
$$

and hence

$$
v \notin \operatorname{Min}_{w} \bigcup_{x \in X} G\left(x, y^{\prime}\right)+C
$$

If $v \in \operatorname{Max} \bigcup_{y \in Y} \operatorname{Min}_{w} \bigcup_{x \in X} G(x, y)$, then, by (iv), we have

$$
v \in \operatorname{Min}_{w} \bigcup_{x \in X} G\left(x, y^{\prime}\right)+C,
$$

which contradicts (4). Therefore, we can deduce the relation $\left(B_{2}\right)$ is valid.

The following example illustrates the validity of Theorem 3.2.

Example 3.2 Let $X=Y=[0,1], C=\mathbb{R}_{+}^{2}, Z=\mathbb{R}^{2}$ and $F(x, y)=\{x\} \times\left\{1-s(y-1)^{2}: s \in[0, x]\right\}$, $G(x, y)=\{x\} \times\left\{1-s(y-1)^{2}: s \in[0,1]\right\}$ for all $(x, y) \in X \times Y$. Then $F(x, y) \subset G(x, y)$ for all $(x, y) \in X \times Y$,

$$
\begin{aligned}
& \bigcup_{x \in X} \operatorname{Max}_{w} \bigcup_{y \in Y} F(x, y)=\{(s, t): 0 \leq s \leq 1,1-s \leq t \leq 1\}, \\
& \operatorname{Min}_{x \in X} \operatorname{Max}_{w} \bigcup_{y \in Y} F(x, y)=\{(s, t): 0 \leq s \leq 1, s+t=1\}
\end{aligned}
$$

and

$$
\operatorname{Max} \bigcup_{y \in Y} \operatorname{Min}_{w} \bigcup_{x \in X} G(x, y)=\{(1,1)\}
$$

We can easily see that the set-valued mappings $F$ and $G$ satisfy all of the continuities in the conditions (i) and (ii) of Theorem 3.2. Let $\Gamma=\left\{g_{1}(x, y), g_{2}(x, y)\right\}$, where $g_{1}(x, y)=$ $-x$ and $g_{2}(x, y)=-y$ for all $(x, y) \in X \times Y$. Let $k=(1,1) \in \operatorname{int} C$ and choose $v=(2,-1) \notin$ $\bigcup_{x \in X} \operatorname{Max}_{w} \bigcup_{y \in Y} F(x, y)+C$. By Corollary 2.4 [10], we have

$$
\xi_{k \nu}(u)=\max _{i=1,2}\left\{g_{i}(u)-g_{i}(v) / g_{i}(k)\right\}=\max \left\{u_{1}-2, u_{2}+1\right\}
$$

for all $u=\left(u_{1}, u_{2}\right) \in Z$. Then $\xi_{k v}(u)>0$ for all $u \in \bigcup_{x \in X} \Lambda(x)$, and

$$
\xi_{k v} F(x, y)=\left\{2-s(y-1)^{2}: 0 \leq s \leq x\right\}
$$

and

$$
\xi_{k v} G(x, y)=\left\{2-s(y-1)^{2}: 0 \leq s \leq 1\right\}
$$

for all $(x, y) \in X \times Y$. 
We claim that the mapping $y \mapsto \xi_{k v} F(x, y)$ is above- $\mathbb{R}_{+}$-concave for each $x \in X$. Indeed, for each $f_{1} \in \xi_{k v} F\left(x, y_{1}\right)$ and $f_{2} \in \xi_{k v} F\left(x, y_{2}\right)$, there exist $s_{1}, s_{2} \in[0, x]$ such that

$$
f_{1}=2-s_{1}\left(y_{1}-1\right)^{2}, \quad f_{2}=2-s_{2}\left(y_{2}-1\right)^{2} .
$$

Then, for each $\lambda \in[0,1]$,

$$
\begin{aligned}
\lambda f_{1}+(1-\lambda) f_{2} & =2-\lambda s_{1}\left(y_{1}-1\right)^{2}-(1-\lambda) s_{2}\left(y_{2}-1\right)^{2} \\
& =2-\left(s_{1} \lambda\left(y_{1}-1\right)^{2}+s_{2}(1-\lambda)\left(y_{2}-1\right)^{2}\right) \\
& \leq 2-s_{3}\left(\lambda y_{1}+(1-\lambda) y_{2}-1\right)^{2} .
\end{aligned}
$$

The last inequality holds by the facts that the mapping $y \mapsto(y-1)^{2}$ is a real-valued convex function and we take $s_{3}=\min \left\{s_{1}, s_{2}\right\}$. Hence $\lambda f_{1}+(1-\lambda) f_{2} \in \xi_{k v} F\left(x, \lambda y_{1}+(1-\lambda) y_{2}\right)-C$ and the mapping $y \mapsto \xi_{k v} F(x, y)$ is above- $\mathbb{R}_{+}$-concave for each $x \in X$. The above-naturally $C$ quasi-convexity for the mapping $x \mapsto G(x, y)$, for each $y \in Y$, can be deduced by a simple calculation, so we leave the proof to the readers.

Furthermore, the condition (iii $\mathrm{i}_{\mathrm{b}}$ ) holds since for each $w \in Y$ and any $x_{w} \in X$, we have $\xi_{k v} G\left(x_{w}, w\right)=\left\{2-s(w-1)^{2}: 0 \leq s \leq 1\right\}$, and hence $\max \xi_{k v} G\left(x_{w}, w\right)=2$. On the other hand, $\max \bigcup_{y \in Y} \min \bigcup_{x \in X} \xi_{k v} G(x, y)=\max \bigcup_{y \in Y} \min \bigcup_{s \in[0,1]}\left\{2-s(y-1)^{2}\right\}=2$. Thus, the condition (iii ${ }_{b}$ ) is valid.

Since $\operatorname{Min}_{w} \bigcup_{x \in X} G(x, w)=\left(\{0\} \times\left[1-(w-1)^{2}, 1\right]\right) \cup\left([0,1] \times\left\{1-(w-1)^{2}\right\}\right)$ for each $w \in Y$, we have

$$
\begin{aligned}
\operatorname{Max} & \bigcup_{y \in Y} \operatorname{Min}_{w} \bigcup_{x \in X} G(x, y) \\
= & \{(1,1)\} \\
& \subset\left(\{0\} \times\left[1-(w-1)^{2}, 1\right]\right) \cup\left([0,1] \times\left\{1-(w-1)^{2}\right\}\right)+C \\
& =\operatorname{Min}_{w} \bigcup_{x \in X} G(x, w)+C
\end{aligned}
$$

for each $w \in Y$. This tells us that condition (iv) of Theorem 3.2 holds.

Therefore, all conditions of Theorem 3.2 hold, and the relation $\left(B_{2}\right)$ is valid since

$$
\begin{aligned}
\operatorname{Max} & \bigcup_{y \in Y} \operatorname{Min}_{w} \bigcup_{x \in X} G(x, y) \\
= & \{(1,1)\} \\
& \subset\{(s, t): 0 \leq s \leq 1,1-s \leq t \leq 1\}+C \\
& =\operatorname{Min} \bigcup_{x \in X} \operatorname{Max}_{w} \bigcup_{y \in Y} F(x, y)+C .
\end{aligned}
$$

The third version of the bilevel minimax theorems is as follows. We remove the condition (iv) in Theorem 3.2 to deduce the relation $\left(B_{3}\right)$. 
Theorem 3.3 Given any Gerstewitz function $\xi_{k v}$ with

$$
v \in \operatorname{Min} \bigcup_{x \in X} \operatorname{Max}_{w} \bigcup_{y \in Y} F(x, y) .
$$

Under the framework of Theorem 3.2 except the condition (iv). Then the relation $\left(B_{3}\right)$ is valid.

Proof For each $x \in X$, let $\Lambda(x)$ be defined the same as in Theorem 3.1. For any $v \in$ $\operatorname{Min} \bigcup_{x \in X} \Lambda(x)$

$$
\left(\bigcup_{x \in X} \Lambda(x) \backslash\{v\}\right) \cap(v-C)=\emptyset .
$$

Then there is a Gerstewitz function $\xi_{k v}: Z \mapsto \mathbb{R}$ with some $k \in \operatorname{int} C$ such that

$$
\xi_{k v}(u)>0
$$

and

$$
\xi_{k v}(v)=0
$$

for all $u \in \bigcup_{x \in X} \Lambda(x) \backslash\{v\}$. Since $\xi_{k v}$ is continuous, by the compactness of $\bigcup_{y \in Y} F(x, y)$, for each $x \in X$, there exist $y_{1} \in Y$ and $f_{1} \in F\left(x, y_{1}\right)$ such that

$$
\xi_{k v}\left(f_{1}\right)=\max \bigcup_{y \in Y} \xi_{k v} F(x, y) .
$$

By Proposition 3.14 [1], $f_{1} \in \operatorname{Max}_{w} \bigcup_{y \in Y} F(x, y)$. Thus, for each $x \in X$, we have

$$
\max \bigcup_{y \in Y} \xi_{k v} F(x, y) \geq 0
$$

or

$$
\min \bigcup_{x \in X} \max \bigcup_{y \in Y} \xi_{k v} F(x, y) \geq 0
$$

From the conditions (i)-(iii) and according to similar arguments in Theorem 3.2, we know that all conditions of Theorem 2.1 hold for the mappings $\xi_{k v} F$ and $\xi_{k \nu} G$. Hence, by Theorem 2.1, we have

$$
\max \bigcup_{y \in Y} \min \bigcup_{x \in X} \xi_{k \nu} G(x, y) \geq 0 .
$$

Since $X$ and $Y$ are compact, there are $x_{0} \in X, y_{0} \in Y$ and $g_{0} \in G\left(x_{0}, y_{0}\right)$ such that

$$
\xi_{k v}\left(g_{0}\right)=\min \bigcup_{x \in X} \xi_{k v} G\left(x, y_{0}\right) \geq 0
$$


Applying Proposition 3.14 in [1], we have $g_{0} \in \operatorname{Min}_{w} \bigcup_{x \in X} G\left(x, y_{0}\right)$. If $g_{0}=v$, we have $v \notin$ $g_{0}+(C \backslash\{0\})$. If $g_{0} \neq v$, we have $\xi_{k v}\left(g_{0}\right)>0$, and hence $g_{0} \notin v-C$. Therefore, $v \notin g_{0}+(C \backslash\{0\})$. Thus, in any case, we have $v \in g_{0}+Z \backslash(C \backslash\{0\})$. This implies that the relation $\left(B_{3}\right)$ is valid.

We illustrate Theorem 3.3 by the following example.

Example 3.3 Let $X, Y, F, G, C, Z, g_{1}, g_{2}, \Gamma$ be given the same as in Example 3.2. Then $F(x, y) \subset G(x, y)$ for all $(x, y) \in X \times Y$ and

$$
\operatorname{Min} \bigcup_{x \in X} \operatorname{Max}_{w} \bigcup_{y \in Y} F(x, y)=\{(t, 1-t): t \in[0,1]\}
$$

Let $k=(1,1) \in \operatorname{int} C$ and choose $v=(1,0) \in \operatorname{Min} \bigcup_{x \in X} \operatorname{Max}_{w} \bigcup_{y \in Y} F(x, y)$. By Corollary 2.4 [10], we have

$$
\xi_{k v}(u)=\max _{i=1,2}\left\{g_{i}(u)-g_{i}(v) / g_{i}(k)\right\}=\max \left\{u_{1}-1, u_{2}\right\}
$$

for all $u=\left(u_{1}, u_{2}\right) \in Z$. Then

$$
\xi_{k v}(u)>0
$$

for all $u \in \bigcup_{x \in X} \operatorname{Max}_{w} \bigcup_{y \in Y} F(x, y) \backslash\{v\}$, and

$$
\xi_{k v} F(x, y)=\left\{1-s(y-1)^{2}: 0 \leq s \leq x\right\}
$$

and

$$
\xi_{k v} G(x, y)=\left\{1-s(y-1)^{2}: 0 \leq s \leq 1\right\}
$$

for all $(x, y) \in X \times Y$.

By a similar discussion in Example 3.2, we know that the mapping $y \mapsto \xi_{k v} F(x, y)$ is above- $\mathbb{R}_{+}$-concave for each $x \in X$, the mapping $x \mapsto G(x, y)$ is above-naturally $C$-quasiconvex for each $y \in Y$ and the condition (iii $i_{\mathrm{b}}$ ) is valid.

Therefore, all conditions of Theorem 3.3 hold, and the relation $\left(B_{3}\right)$ is valid since

$$
\begin{aligned}
& \operatorname{Min} \bigcup_{x \in X} \operatorname{Max}_{w} \bigcup_{y \in Y} F(x, y) \\
& =\{(t, 1-t): t \in[0,1]\} \\
& \subset\{(1,1)\}+Z \backslash(C \backslash\{0\}) \\
& =\operatorname{Max} \bigcup_{y \in Y} \operatorname{Min}_{w} \bigcup_{x \in X} G(x, y)+Z \backslash(C \backslash\{0\}) .
\end{aligned}
$$

Remark 3.2 We note that Theorems 3.2-3.3 include the case $G=F$ as a special case. 


\section{Competing interests}

The author declares that they have no competing interests.

\section{Acknowledgements}

This work was supported by 'Department of Occupational Safety and Health, College of Public Health, China Medical University, Taiwan' that are gratefully acknowledged. The author would like to thank the editor and the reviewers for their valuable comments and suggestions to improve this paper.

Received: 17 January 2014 Accepted: 23 April 2014 Published: 12 May 2014

\section{References}

1. Lin, YC, Ansari, QH, Lai, HC: Minimax theorems for set-valued mappings under cone-convexities. Abstr. Appl. Anal. (2012) doi:10.1155/2012/310818

2. Li, SJ, Chen, GY, Teo, KL, Yang, XQ: Generalized minimax inequalities for set-valued mappings. J. Math. Anal. Appl. 281, 707-723 (2003)

3. Li, SJ, Chen, GY, Lee, GM: Minimax theorems for set-valued mappings. J. Optim. Theory Appl. 106, 183-199 (2000)

4. Ferro, F: Optimization and stability results through cone lower semi-continuity. Set-Valued Anal. 5, 365-375 (1997)

5. Ha, CW: Minimax and fixed-point theorems. Math. Ann. 248, 73-77 (1980)

6. Berge, C: Topological Spaces. Macmillan Co., New York (1963)

7. Aubin, JP, Cellina, A: Differential Inclusions. Springer, Berlin (1984)

8. Gerth, C, Weidner, P: Nonconvex separation theorems and some applications in vector optimization. J. Optim. Theory Appl. 67, 297-320 (1990)

9. Tan, KK, Yu, J, Yuan, XZ: Existence theorems for saddle points of vector-valued maps. J. Optim. Theory Appl. 89, 731-747 (1996)

10. Chen, GY, Goh, CJ, Yang, XQ: Vector network equilibrium problems and nonlinear scalarization methods. Math. Methods Oper. Res. 49, 239-253 (1999)

10.1186/1029-242X-2014-182

Cite this article as: Lin: Bilevel minimax theorems for non-continuous set-valued mappings. Journal of Inequalities and Applications 2014, 2014:182

\section{Submit your manuscript to a SpringerOpen ${ }^{\circ}$ journal and benefit from:}

- Convenient online submission

- Rigorous peer review

Immediate publication on acceptance

- Open access: articles freely available online

- High visibility within the field

- Retaining the copyright to your article 